\title{
Ambulatory surgery for the patient with breast cancer: current perspectives
}

\author{
Chong Han Pek' \\ John Tey² \\ Ern Yu Tan' \\ 'Department of General Surgery, \\ ${ }^{2}$ Department of Anaesthesiology, \\ Intensive Care and Pain Medicine, \\ Tan Tock Seng Hospital, Singapore, \\ Singapore
}

This article was published in the following Dove Press journal:

Open Access Surgery

16 August 2016

Number of times this article has been viewed
Correspondence: Ern Yu Tan Department of General Surgery, Tan Tock Seng Hospital, II Jalan Tan Tock Seng, Singapore 308433 , Singapore

$\mathrm{Tel}+6563577807$

Fax +65 63577809

Email Ern_Yu_Tan@ttsh.com.sg
Abstract: Ambulatory breast cancer surgery is well accepted and is the standard of care at many tertiary centers. Rather than being hospitalized after surgery, patients are discharged on the day of surgery or within 23 hours. Such early discharge does not adversely affect patient outcomes and has the added benefits of better psychological adjustment for the patient, economic savings, and a more efficient utilization of health care resources. The minimal care needed post-discharge also means that the caregiver is not unduly burdened. Unplanned conversions to inpatient admission and readmission rates are low. Wound complications are infrequent and no issues with drain care have been reported. Because the period of postoperative observation is short and monitoring is not as intensive, ambulatory surgery is only suitable for low-risk procedures such as breast cancer surgery and in patients without serious comorbidities, where the likelihood of major perioperative events is low. Optimal management of pain, nausea, and vomiting is essential to ensure a quick recovery and return to normal function. Regional anesthesia such as the thoracic paravertebral block has been employed to improve pain control during the surgery and in the immediate postoperative period. The block provides excellent pain relief and reduces the need for opiates, which also consequently reduces the incidence of nausea and vomiting. The increasing popularity of total intravenous anesthesia with propofol has also helped reduce the incidence of nausea and vomiting in the postoperative period. Ambulatory surgery can be safely carried out in centers where there is a well-designed workflow to ensure proper patient selection, counseling, and education, and where patients and caregivers have easy access to medical services should problems arise after discharge.

Keywords: ambulatory surgery, early discharge, breast cancer

\section{Introduction}

Ambulatory surgery is now commonplace in many tertiary centers and has been embraced as a safe and economical alternative to inpatient admission. Patients are either discharged the same day of surgery or within 23 hours (ambulatory surgery, AS23), and therefore a fast recovery from anesthesia and return to normal function are necessary. Since the period of postoperative observation is short and monitoring is not as intensive, ambulatory surgery is suitable only for those without serious preexisting conditions and for surgical procedures with a low risk of postoperative complications. Breast cancer surgery is increasingly being performed in an ambulatory setting at many tertiary centers. Early discharge does not compromise surgical outcomes ${ }^{1,2}$ and has even been reported to benefit patients psychologically., Ambulatory surgery units allow a more efficient utilization of health care resources and are particularly advantageous in centers with high caseloads, where a quick patient turnover is crucial in alleviating 
the pressure on inpatient bed occupancy. Many ambulatory centers, in fact, function outside of a hospital facility.

Breast cancer surgery is well suited for ambulatory surgery. The thoracic and abdominal cavities are not breached, and dissection is away from the airway and major vessels, thus entailing a low risk of major perioperative complications and intensive postoperative monitoring is seldom required. Most patients are able to resume diet and ambulate within a few hours of surgery. Advances in surgical techniques have made breast cancer surgery suitable as an ambulatory procedure. Resection has become less extensive over the years and modified radical mastectomy, where only the breast and overlying skin are removed, is the current standard of care. The Halstead radical mastectomy, where the underlying pectoralis muscles are also resected, is now rarely performed. Even large locally advanced tumors that invade the chest wall often respond well enough to neoadjuvant chemotherapy and radiation for chest wall resection to be avoided. ${ }^{5}$ Preservation of the pectoralis muscles dramatically reduces the surgical morbidity; the potential for postoperative complications is reduced, postoperative pain is less severe, and ipsilateral arm movements are less affected. Neoadjuvant chemotherapy has also been employed to downsize operable tumors in order to facilitate breast-conserving surgery (wide local excision, WLE) in cases where mastectomy would otherwise have been necessary. The main advantage of WLE, in relation to early discharge, is that there is minimal risk of postoperative bleeding and seroma formation and, consequently, patients can be discharged after a short period of postoperative observation without any surgical drains. The surgical wound requires minimal care, and wound site pain is often mild and manageable with simple oral analgesia. Nevertheless, oncoplastic techniques that have evolved in the past decade are increasingly being incorporated into WLE to allow more extensive resections and to achieve a superior cosmetic outcome. ${ }^{6,7}$ Such techniques can involve extensive mobilization of the breast plates to reshape the breast, and can even involve volume replacement with autologous tissue flaps, and are often not suitable as ambulatory procedures. Newer modalities, such as intraoperative radiotherapy following WLE for low-risk cancers, however, have been associated with minimal morbidity and do not compromise the suitability for ambulatory surgery. ${ }^{89}$ Nodal dissection techniques have become less extensive. The adoption of sentinel lymph node (SLN) biopsy as the standard of care in the 2000s more than halved the rate of full axillary lymph node dissection. ${ }^{10-12}$ False-negative rates are low and a negative SLN is now accepted as being representative of node-negative disease. ${ }^{13-15}$ The recent American College of
Surgeons Oncology Group Z0011 trial has further shown that axillary lymph node dissection can be omitted in women with low-burden SLN disease who receive adjuvant whole breast irradiation and chemotherapy. ${ }^{16}$ Since SLN biopsy involves only a limited dissection of the low level I axilla, potential complications of postoperative bleeding from a slipped clip or ligature, or oozing from the surgical bed and seroma formation is minimal, obliterating the need for surgical drains. This increases patient and caregiver confidence for early discharge and consequently women undergoing WLE and SLN biopsy are most often discharged the same day of surgery.

Many patients undergoing breast cancer surgery in our center are discharged the morning after the surgery (within 23 hours, AS23). Discharge after overnight observation is more easily accepted than same-day discharge, and both patient and caregivers feel more reassured with specialized care in a hospital setting during the immediate postoperative period. One study reported that $40 \%$ of patients would have preferred to stay overnight after surgery, rather than be discharged the same day. ${ }^{3}$ This option of an overnight stay reduces inpatient conversion rates and is especially advantageous in those who take longer to recover from postoperative nausea, vomiting, or giddiness, and those with medical conditions such as severe obstructive sleep apnea and morbid obesity who require a longer period of observation. From a logistics standpoint, the AS23 option also makes it easier to manage operating lists and avoids the need to convert to inpatient admission when the surgeries end too late for same-day discharge. This is particularly relevant to centers like ours where limited operating room resources mean that breast cancer surgeries and minor nonbreast surgeries, such as simple excision biopsies, are scheduled on the same operating list; excision biopsies are typically listed first so that patients have adequate time to recover from anesthesia and be discharged the same day. Consequently, patients undergoing breast cancer surgery often come out of surgery later in the afternoon or even in the evening, and an overnight stay is almost inevitable.

Fundamental to the success of ambulatory surgery is a multidisciplinary approach for proper patient selection, counseling, and education on postsurgical care. Breast cancer surgeries, apart from immediate autologous flap reconstruction and more extensive oncoplastic procedures, are low risk in nature, cause minimal impairment of physiological function, and are unlikely to be associated with serious postoperative events. Moreover, the majority of patients undergoing such surgeries are relatively healthy, ${ }^{17}$ and a short period of observation with basic monitoring is not expected to compromise patient safety or outcome. Whereas in the 
past where ambulatory surgery was largely limited to patients with American Society of Anesthesiologists status of 1 or 2, experience and studies have shown that even American Society of Anesthesiologists status 3 patients can safely undergo ambulatory surgery so long as their systemic diseases are well controlled. ${ }^{17}$ Likewise, physical age in itself is no longer an exclusion factor. ${ }^{18}$ Patients are generally screened by the surgeon and then evaluated by an anesthetist, either prior to or on the day of surgery itself. Anesthetic evaluation prior to the day of surgery has several benefits. Patient expectations and anxiety regarding the surgery and anesthesia can be better managed, and importantly, patients at risk of perioperative events and who would require more intensive monitoring can be identified and excluded from the ambulatory setting. These would include those with high cardiac risk, who may require serial cardiac assessment, as well as those on anticoagulation, who are at risk of significant postoperative bleeding. Patients with uncontrolled systemic diseases and those with undiagnosed medical conditions can also be identified and referred for optimization prior to surgery, which can generally be safely postponed until the patient is medically stable.

The use of short-acting anesthetic agents that allow rapid emergence and recovery from anesthesia has reduced the time needed for close monitoring in the immediate postoperative period. While it remains the practice in many ambulatory centers that patients are transferred from the operating room to the postanesthesia recovery care unit for intensive monitoring immediately after surgery, ${ }^{19}$ one prospective study involving 5,000 patients reported that the postanesthesia recovery care unit could be safely bypassed in more than $50 \%$ of patients, without any increase in complications or delay in planned discharge. ${ }^{20}$ Optimal control of postoperative nausea, vomiting, and pain are essential for early discharge. Pain should be manageable with simple oral analgesia and patients should be able to eat and ambulate without or with only minimal assistance by the time of discharge. However, adequate home and social support is still needed because of wound and drain care issues and some restriction of shoulder and arm movements, especially in those with axillary clearance. Caregiver education is essential, and clear instructions are necessary to ensure prompt and easy access to medical services should problems develop after the discharge. Specialist breast care nurses play a key role in these aspects. ${ }^{21}$ They provide preoperative counseling to help allay fears and anxiety related to the diagnosis and the surgery, educate the patient and caregivers on wound and drain care and arm exercises; these are later reinforced during the review prior to discharge from the ambulatory center. This process is important in boosting patient and caregiver confidence for early discharge and reduces the conversion to inpatient admission as well as readmission rates.

The majority of breast cancer surgeries are now performed as ambulatory procedures. After the ambulatory center was established at our hospital in 2004 , more than $70 \%$ of breast cancer surgeries were performed as ambulatory procedures. ${ }^{22}$ Those undergoing immediate breast reconstruction, who require intensive postoperative monitoring, and those without any home support, including institutionalized patients, continue to require inpatient admission. The large majority of patients were discharged according to plan. ${ }^{21} \mathrm{~A}$ small number of patients required conversion to inpatient admission, most often for unresolved postoperative symptoms such as nausea and vomiting, wound-related problems, or unanticipated perioperative events. However, most of these resolved with expectant management and the patients were discharged soon after. ${ }^{22}$ Readmission was uncommon and most studies quote rates of between $0 \%$ and $1 \% .^{2,23,24}$ We previously reported a $3.6 \%$ readmission rate after ambulatory surgery (including patients discharged within 23 hours), with wound-related problems such as infection and hematoma being the reason for readmission in many instances. ${ }^{22}$ But, in spite of this, overall wound complications were not reportedly more frequent after ambulatory surgery. ${ }^{21}$ Specifically, the incidence of postoperative bleeding was low and wound exploration for hemostasis was necessary in less than half the cases; many of these unplanned explorations took place on the same day of surgery and did not delay the planned discharge. ${ }^{22}$ Wound infection rates were also similar and implied that home care of the wound and drains is adequate.

Adequate control of pain, nausea, and vomiting are important in the postoperative period not only because they are in themselves distressing to the patient, but also because they impede the return of normal activities and can delay discharge from the ambulatory center. ${ }^{25}$ In breast surgery, postoperative pain management usually consists of a combination of oral analgesics, including acetaminophen, opiates such as panadeine and tramadol, and nonsteroidal anti-inflammatory agents. More recently, regional nerve blocks with long-acting local anesthetic agents (bupivacaine, ropivacaine), often combined with epinephrine, have been employed as an adjunct to general anesthesia. These are highly effective in pain control and reduce the need for opiates. ${ }^{26}$ Regional anesthesia can be administered as a single-shot thoracic paravertebral block (PVB), either at a single level (at the level of T3) or at multiple levels from thoracic interspace 1 through to $5 .^{26}$ These are often sufficient to last the entire duration of the surgery, though top-up analgesics may be needed to supplement pain 
control. Repeated dosing is possible with the use of thoracic interspace catheters (inserted into the ipsilateral paravertebral space at the level of $\mathrm{T} 2 / 3$ or T3/4) and allow analgesia to be maintained during long surgeries and into the postoperative period; catheters are removed prior to discharge. ${ }^{27}$ PVBs are generally administered under ultrasound guidance and complications are rare, even with the use of catheters. The use of a test dose prior to injection of the full anesthetic dose reduces the risk of anaphylaxis, and other potential complications such as inadvertent lung or vessel injury, and lidocaine toxicity will manifest during the block or surgery and are unlikely to present in the postoperative period. Several centers have reported improved patient outcomes with the addition of PVB. ${ }^{28}$ Pain scores at 1 and 6 hours after surgery were significantly lower in those receiving PVB, and rescue analgesics were less often needed, translating into earlier discharge and greater patient satisfaction. ${ }^{29-31}$ Postoperative pain control with regional blocks was reportedly superior to systemic analgesia. ${ }^{32}$ Pain control in the immediate postoperative period may even have long-term consequences. The degree of acute pain has been correlated with the development and intensity of chronic pain, ${ }^{33,34}$ which can affect up to one-third of patients after breast surgery. ${ }^{35}$

Apart from superior pain control, PVB helps also to reduce postoperative nausea and vomiting (PONV), likely a direct result of reduced opiate use. ${ }^{29} \mathrm{PONV}$ is particularly relevant to breast cancer surgery since patients are mostly female, which in itself is an independent predictor of PONV. ${ }^{36}$ Anti-emetics, such as dexamethasone and ondansetron, are the main agents used to manage PONV and are given prophylactically. A switch to total intravenous anesthesia (TIVA), where an intravenous drug (propofol) is used to both induce and maintain anesthesia, instead of inhalational agents, has also helped to reduce PONV. ${ }^{37}$ Increasingly, TIVA is being used in ambulatory procedures because reversal is more rapid and predictable, as propofol is short-acting and its pharmacokinetic properties are well understood. In addition, with the use of target-controlled infusion systems, the depth of anesthesia can be titrated accordingly by changing the desired effect site or plasma concentration on the target-controlled infusion device, which then calculates and adjusts the propofol infusion rates according to the drug pharmacokinetics. ${ }^{38}$

Moving forward, the use of TIVA and PVB can enhance postoperative recovery and can potentially increase the rate of same-day discharge after more extensive procedures such as mastectomy and axillary clearance. Another aspect that is being evaluated is the need for surgical drains. Closed suction drains give an early indication of significant postoperative bleeding and are effective in reducing seroma formation, which may affect wound healing. Early discharge means that patients may need to manage one or two drains at home. Drain care is minimal and the soft silicone drains with a small hub used cause little discomfort and do not interfere much with daily activities. Even so, it would be ideal to discharge patients without any drains in situ. A meta-analysis concluded that early drain removal increased the incidence of seroma formation, but did not find any significant differences in wound-healing rates or wound complications. ${ }^{39}$ More recent studies, however, have reported otherwise and at least one study omitted surgical drains completely. ${ }^{40-42}$ Repeated aspirations may be more frequent when drains are removed early or not used at all, but the standard practice of removing drains only when the drainage has decreased significantly does not completely eliminate seroma re-accumulation after drain removal and patients may still require aspiration. Numerous methods have been evaluated to reduce seroma formation, though the effect has been modest at best. Energy devices such as electrocautery are widely used in dissection and help seal small lymphatic channels that are inevitably transected. Newer energy devices such as laser scalpel, argon diathermy, ultrasonic scalpel, ultrasonic scissors, and vessel sealing systems have been evaluated with varying results and have not been conclusively shown to be superior to electrocautery. ${ }^{43}$ Fibrin sealants, such as fibrin glue, were postulated to reduce exudates from acute inflammatory reactions induced by the surgery, and though initial results were promising, these were not reproduced in subsequent prospective trials. ${ }^{44,45}$ Yet another approach involves intraoperative surgical quilting to reduce the resultant dead space, particularly after mastectomy and axillary clearance, though further studies are needed to define the extent of benefit, as quilting inevitably increases the operative time. ${ }^{46,47}$

Ambulatory breast cancer surgery has been proven safe in centers with a well-defined infrastructure that ensures proper patient selection, counseling, and education and where there is easy access to medical services after discharge. Newer modalities such as regional anesthetic blocks and total intravenous anesthesia help improve postoperative recovery and can potentially increase the rate of same-day discharge. Although postoperative drains remain a common practice following mastectomy and axillary clearance, some have questioned its benefit. Drains may reduce the incidence of seroma formation, which requires repeated aspirations, but this may not otherwise hasten complete resolution or wound healing. Apart from the economic benefits ${ }^{2}$ and the more efficient use of health care resources, ambulatory surgery is also well accepted by patients and their caregivers. ${ }^{24,48,49}$ Patients being discharged early have reported better psychological adjustment and resume normal 
daily activities fairly quickly. ${ }^{1,3}$ The burden on the caregivers is also minimal..$^{50}$ In fact, majority of the patients responded favorably when asked about their experience and indicated that they would be happy to undergo ambulatory surgery again..$^{24}$

\section{Disclosure}

The authors report no conflicts of interest in this work.

\section{References}

1. Dooley WC. Ambulatory breast cancer surgery. Ann Surg Oncol. 2000;7(3):174-175.

2. Goodman AA, Mendez AL. Definitive surgery for breast cancer performed on an outpatient basis. Arch Surg. 1993;128(10):1149-1152.

3. Margolese RG, Lasry JC. Ambulatory surgery for breast cancer patients. Ann Surg Oncol. 2000;7(3):181-187.

4. McManus SA, Topp DA, Hopkins C. Advantages of outpatient breast surgery. Am Surg. 1994;60(12):967-970.

5. Thompson AM, Moulder-Thompson SL. Neoadjuvant treatment of breast cancer. Ann Oncol. 2012;23 (Suppl 10):x231-x236.

6. Yang JD, Lee JW, Cho YK, et al. Surgical techniques for personalized oncoplastic surgery in breast cancer patients with small- to moderate-sized breasts (part 1): volume displacement. J Breast Cancer. 2012;15(1):1-6.

7. Clough KB, Lewis JS, Couturaud B, Fitoussi A, Nos C, Falcou MC. Oncoplastic techniques allow extensive resections for breast-conserving therapy of breast carcinomas. Ann Surg. 2003;237(1):26-34.

8. Eldredge-Hindy HB, Rosenberg AL, Simone NL. Intraoperative radiotherapy for breast cancer: the lasting effects of a fleeting treatment. Int $J$ Breast Cancer. 2014;2014:214325.

9. Tuschy B, Berlit S, Romero S, et al. Clinical aspects of intraoperative radiotherapy in early breast cancer: short-term complications after IORT in women treated with low energy x-rays. Radiat Oncol. 2013;8:95.

10. Chen AY, Halpern MT, Schrag NM, Stewart A, Leitch M, Ward E. Disparities and trends in sentinel lymph node biopsy among early-stage breast cancer patients (1998-2005). J Natl Cancer Inst. 2008;100(7):462-474.

11. Quan ML, Hodgson N, Lovrics P, Porter G, Poirier B, Wright FC. National adoption of sentinel node biopsy for breast cancer: lessons learned from the Canadian experience. Breast J. 2008;14(5):421-427.

12. Heneghan HM, Prichard RS, Devaney A, et al. Evolution of breast cancer management in Ireland: a decade of change. BMC Surg. 2009;9:15.

13. Veronesi U, Viale G, Paganelli G, et al. Sentinel lymph node biopsy in breast cancer: ten-year results of a randomized controlled study. Ann Surg. 2010;251(4):595-600.

14. Krag DN, Anderson SJ, Julian TB, et al. Sentinel-lymph-node resection compared with conventional axillary-lymph-node dissection in clinically node-negative patients with breast cancer: overall survival findings from the NSABP B-32 randomised phase 3 trial. Lancet Oncol. 2010;11(10):927-933.

15. Ang CH, Tan MY, Teo C, et al. Blue dye is sufficient for sentinel lymph node biopsy in breast cancer. Br J Surg. 2014;101(4):383-389; discussion 389

16. Giuliano AE, Hunt KK, Ballman KV, et al. Axillary dissection vs no axillary dissection in women with invasive breast cancer and sentinel node metastasis: a randomized clinical trial. JAMA. 2011;305(6):569-575.

17. Natof HE. Pre-existing medical problems. Ambulatory surgery. IMJ Ill Med J. 1984;166(2):101-104.

18. Chung F, Mezei G, Tong D. Pre-existing medical conditions as predictors of adverse events in day-case surgery. Br JAnaesth. 1999;83(2):262-270.

19. Chung F. Recovery pattern and home-readiness after ambulatory surgery. Anesth Analg. 1995;80(5):896-902.

20. Apfelbaum JL, Walawander CA, Grasela TH, et al. Eliminating intensive postoperative care in same-day surgery patients using short-acting anesthetics. Anesthesiology. 2002;97(1):66-74.

21. Marla S, Stallard S. Systematic review of day surgery for breast cancer. Int J Surg. 2009;7(4):318-323.
22. Ng YY, Chan PM, Chen JJ, Seah MD, Teo C, Tan EY. Adopting ambulatory breast cancer surgery as the standard of care in an Asian population. Int J Breast Cancer. 2014;2014:672743.

23. Mezei G, Chung F. Return hospital visits and hospital readmissions after ambulatory surgery. Ann Surg. 1999;230(5):721-727.

24. Marchal F, Dravet F, Classe JM, et al. Post-operative care and patient satisfaction after ambulatory surgery for breast cancer patients. Eur $J$ Surg Oncol. 2005;31(5):495-499.

25. Pavlin DJ, Chen C, Penaloza DA, Buckley FP. A survey of pain and other symptoms that affect the recovery process after discharge from an ambulatory surgery unit. J Clin Anesth. 2004;16(3):200-206.

26. Kairaluoma PM, Bachmann MS, Korpinen AK, Rosenberg PH, Pere PJ. Single-injection paravertebral block before general anesthesia enhances analgesia after breast cancer surgery with and without associated lymph node biopsy. Anesth Analg. 2004;99(6):1837-1843.

27. Karmakar MK. Thoracic paravertebral block. Anesthesiology. 2001;95(3): 771-780.

28. Klein SM, Bergh A, Steele SM, Georgiade GS, Greengrass RA. Thoracic paravertebral block for breast surgery. Anesth Analg. 2000;90(6):1402-1405.

29. Tahiri Y, Tran de QH, Bouteaud J, et al. General anaesthesia versus thoracic paravertebral block for breast surgery: a meta-analysis. J Plast Reconstr Aesthet Surg. 2011;64(10):1261-1269.

30. Schnabel A, Reichl SU, Kranke P, Pogatzki-Zahn EM, Zahn PK. Efficacy and safety of paravertebral blocks in breast surgery: a meta-analysis of randomized controlled trials. Br J Anaesth. 2010;105(6):842-852.

31. Moller JF, Nikolajsen L, Rodt SA, Ronning H, Carlsson PS. Thoracic paravertebral block for breast cancer surgery: a randomized double-blind study. Anesth Analg. 2007;105(6):1848-1851.

32. Block BM, Liu SS, Rowlingson AJ, Cowan AR, Cowan JA, Jr., Wu CL. Efficacy of postoperative epidural analgesia: a meta-analysis. JAMA. 2003;290(18):2455-2463.

33. Tasmuth T, Kataja M, Blomqvist C, von Smitten K, Kalso E. Treatmentrelated factors predisposing to chronic pain in patients with breast cancer--a multivariate approach. Acta Oncol. 1997;36(6):625-630.

34. Obata H, Saito S, Fujita N, Fuse Y, Ishizaki K, Goto F. Epidural block with mepivacaine before surgery reduces long-term post-thoracotomy pain. Can J Anaesth. 1999;46(12):1127-1132.

35. Iohom G, Abdalla H, O'Brien J, et al. The associations between severity of early postoperative pain, chronic postsurgical pain and plasma concentration of stable nitric oxide products after breast surgery. Anesth Analg. 2006;103(4):995-1000.

36. Apfel CC, Philip BK, Cakmakkaya OS, et al. Who is at risk for postdischarge nausea and vomiting after ambulatory surgery? Anesthesiology. 2012;117(3):475-486.

37. Eikaas H, Raeder J. Total intravenous anaesthesia techniques for ambulatory surgery. Curr Opin Anaesthesiol. 2009;22(6):725-729.

38. Struys MM, Sahinovic M, Lichtenbelt BJ, Vereecke HE, Absalom AR. Optimizing intravenous drug administration by applying pharmacokinetic/pharmacodynamic concepts. Br J Anaesth. 2011;107(1):38-47.

39. Droeser RA, Frey DM, Oertli D, et al. Volume-controlled vs no/shortterm drainage after axillary lymph node dissection in breast cancer surgery: a meta-analysis. Breast. 2009;18(2):109-114.

40. Taylor JC, Rai S, Hoar F, Brown H, Vishwanath L. Breast cancer surgery without suction drainage: the impact of adopting a 'no drains' policy on symptomatic seroma formation rates. Eur J Surg Oncol. 2013;39(4):334-338.

41. Troost MS, Kempees CJ, de Roos MA. Breast cancer surgery without drains: no influence on seroma formation. Int J Surg. 2015;13:170-174.

42. Okada N, Narita Y, Takada M, et al. Early removal of drains and the incidence of seroma after breast surgery. Breast Cancer. 2015;22(1):79-83.

43. Srivastava V, Basu S, Shukla VK. Seroma formation after breast cancer surgery: what we have learned in the last two decades. J Breast Cancer. 2012;15(4):373-380.

44. van Bemmel AJ, van de Velde CJ, Schmitz RF, Liefers GJ. Prevention of seroma formation after axillary dissection in breast cancer: a systematic review. Eur J Surg Oncol. 2011;37(10):829-835. 
45. Sajid MS, Hutson K, Kalra L, Bonomi R. The role of fibrin glue instillation under skin flaps in the prevention of seroma formation and related morbidities following breast and axillary surgery for breast cancer: a meta-analysis. J Surg Oncol. 2012;106(6): 783-795.

46. ten Wolde B, van den Wildenberg FJ, Keemers-Gels ME, Polat F, Strobbe LJ. Quilting prevents seroma formation following breast cancer surgery: closing the dead space by quilting prevents seroma following axillary lymph node dissection and mastectomy. Ann Surg Oncol. 2014;21(3):802-807.
47. Kottayasamy Seenivasagam R, Gupta V, Singh G. Prevention of seroma formation after axillary dissection--a comparative randomized clinical trial of three methods. Breast J. 2013;19(5):478-484.

48. Dooley WC. Ambulatory mastectomy. Am J Surg. 2002;184(6): 545-548; discussion 548-549.

49. Marrazzo A, Taormina P, David M, et al. Surgical treatment of early breast cancer in day surgery. Chir Ital. 2007;59(5):687-691.

50. Wells M, Harrow A, Donnan P, et al. Patient, carer and health service outcomes of nurse-led early discharge after breast cancer surgery: a randomised controlled trial. Br J Cancer. 2004;91(4):651-658.
Open Access Surgery

\section{Publish your work in this journal}

Open Access Surgery is an international, peer-reviewed, open access journal that focuses on all aspects of surgical procedures and interventions. Patient care around the peri-operative period and patient outcomes post surgery are key topics for the journal. All grades of surgery from minor cosmetic interventions to major surgical procedures are covered. Novel techniques and the
Dovepress

utilization of new instruments and materials, including implants and prostheses that optimize outcomes constitute major areas of interest. The manuscrip management system is completely online and includes a very quick and fair peer-review system, which is all easy to use. Visit http://www.dovepress.com/ testimonials.php to read real quotes from published authors. 